\title{
Modulation of bone morphogenetic protein signaling inhibits the onset and progression of ankylosing enthesitis
}

\author{
Rik J.U. Lories, Inge Derese, and Frank P. Luyten \\ Laboratory for Skeletal Development and Joint Disorders, Department of Rheumatology, University Hospitals Leuven, \\ Katholieke Universiteit Leuven, Leuven, Belgium.
}

\begin{abstract}
Joint ankylosis is a major cause of disability in the human spondyloarthropathies. Here we report that this process partially recapitulates embryonic endochondral bone formation in a spontaneous model of arthritis in DBA/1 mice. Bone morphogenetic protein (BMP) signaling appears to be a key molecular pathway involved in this pathological cascade. Systemic gene transfer of noggin, a BMP antagonist, is effective both as a preventive and a therapeutic strategy in the mouse model, mechanistically interfering with enthesial progenitor cell proliferation in early stages of the disease process. Immunohistochemical staining for phosphorylated smad1/5 in enthesial biopsies of patients with spondyloarthropathy reveals active BMP signaling in similar target cells. Our data suggest that BMP signaling is an attractive therapeutic target for interfering with structural changes in spondyloarthropathy either as an alternative or complementary approach to current antiinflammatory treatments.
\end{abstract}

\section{Introduction}

The human spondyloarthropathies are a group of chronic inflammatory joint disorders with a prevalence of about $0.3 \%$ (1), which primarily affect individuals between 20 and 40 years of age. Different diagnostic entities, based on clinical, genetic, and pathological characteristics, are classified within this disease concept (2). These consist of ankylosing spondylitis, psoriatic arthritis, reactive arthritis, arthritis associated with inflammatory bowel disease, and a juvenile and an undifferentiated form of arthritis. Clinical manifestations include arthritis and ankylosis, which cause severe and permanent disability. Increasing evidence suggests that an anatomical zone referred to as the enthesis, where tendons and ligaments attach to underlying bone, is a primary target of the pathological process $(3,4)$. Enthesitis, synovitis, and subchondral bone changes are associated with enthesial cell proliferation and heterotopic cartilage and bone formation, all of which eventually lead to joint space bridging, a process called ankylosing enthesitis. Together, these features are considered the hallmark of spondyloarthropathy (3-5).

Long-term treatment strategies for chronic joint diseases aim to prevent tissue damage and loss of joint function. So-called disease modifying treatments not only target symptom control but are also intended to influence the pathological process itself. In spondyloarthropathy, conventional therapies, such as nonsteroidal antiinflammatory drugs and immune modulators, are considered symptom-controlling rather than disease-modifying treatments (6). TNF inhibitory agents have been introduced as biologic response modifiers $(7,8)$. Despite their clinical efficacy and widespread use, and in contrast to what is seen in rheumatoid arthritis, the disease-

Nonstandard abbreviations used: BMP, bone morphogenetic protein; PCNA, proliferating cell nuclear antigen; Sox9, sex-determining region Y-box 9; TBS, Tris-buffered saline.

Conflict of interest: The authors have declared that no conflict of interest exists. However, Rik Lories and Frank P. Luyten have submitted a patent application regarding the use of BMP inhibition in spondyloarthropathy.

Citation for this article: J. Clin. Invest. 115:1571-1579 (2005).

doi:10.1172/JCI23738. modifying properties of these strategies remain unclear. In chronic joint diseases such as rheumatoid arthritis and in animal models of these diseases (9), inflammation is associated with bone loss and destruction. In spondyloarthropathy, new bone formation is characteristic. Coupling of inflammation and subsequent ankylosis have not been demonstrated. Progressive ankylosis may persist despite absence of clinical disease activity (10). It is possible that continued suppression of inflammation may result in accelerated bone formation and ankylosis once the pathological cascade has been triggered. Therapeutic strategies specifically targeting cartilage and bone formation may therefore be required, either as an alternative or a complementary approach to immune-suppressing drugs, for gaining full control of the disease (11).

Bone morphogenetic proteins (BMPs), discovered to be proteins that ectopically induce a cascade of endochondral bone formation (12), play a crucial role in skeletal $(13,14)$ and joint morphogenesis (15-18). BMPs are members of the TGF- $\beta$ superfamily, a group of polypeptides capable of regulating a wide array of cellular processes such as proliferation, differentiation, lineage determination, motility, and death $(19,20)$. In their canonical pathway, BMPs induce ligand-dependent type I and type II receptor heterodimerization, leading to phosphorylation of smad-signaling molecules (smad1/5) that bind smad 4 and then translocate to the nucleus. BMP signaling is controlled at many levels, including that of extracellular antagonists such as noggin (19). Reactivation of embryonic-signaling pathways has been suggested as an essential part of repair and homeostasis in health and disease $(21,22)$. However, untimely or unwanted activation of signaling cascades fundamental for normal development may promote disease processes such as spondyloarthropathies. Therefore, we hypothesized that BMP signaling could play a direct role in joint ankylosis.

To address this, we studied BMP signaling in a spontaneous model of arthritis. Grouped caging of male DBA/1 mice led to the development of arthritis affecting hind paws and characterized by ankylosing enthesitis (23-25). This feature clearly distinguishes the model from other animal models of different types of joint 


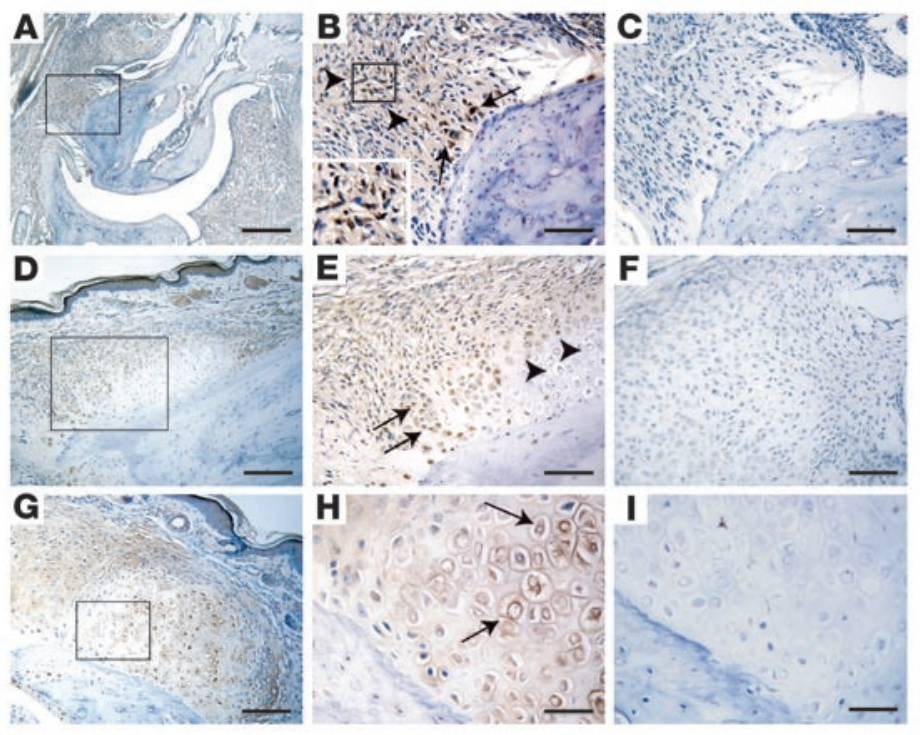

\section{Figure 1}

BMP immunohistochemistry in ankylosing enthesitis. (A-C) The first phase of ankylosing enthesitis is characterized by enthesial cell proliferation and cartilage differentiation; immunoreactivity for BMP2 is detected in spindle-shaped enthesial fibroblast-like cells (arrowheads, inset in $\mathbf{B}$ ) and in more rounded early chondroblast-like cells (arrows) (A; $\mathbf{B}$, detail of boxed area in A). At this stage of the disease process, no cartilage hypertrophy is seen. (C) Negative control staining using IgG. (D-I) Cartilage formation in ankylosing enthesitis shows BMP7-positive proliferating and prehypertrophic chondrocyte-like cells (D; E, detail of boxed area in $\mathbf{D}$; arrows indicate zone of positive cells) and BMP6-positive hypertrophic chondrocyte-like cells (G; H, detail of boxed area in G; arrows indicate positive cells). No BMP7 expression is seen in hypertrophic chondrocyte-like cells $(E$, arrowheads). ( $\mathbf{F}$ and $\mathbf{I})$ Negative control staining using IgG. Scale bars: $200 \mu \mathrm{m}$ in $\mathbf{A}$ and $\mathbf{D} ; 100 \mu \mathrm{m}$ in $\mathbf{G} ; 80 \mu \mathrm{m}$ in $\mathbf{E}$ and $\mathbf{F} ; 50 \mu \mathrm{m}$ in $\mathbf{B}$ and $\mathbf{C} ; 25 \mu \mathrm{m}$ in $\mathbf{H}$ and $\mathbf{I}$. diseases, such as rheumatoid arthritis and osteoarthritis, that are characterized by either synovial inflammation or progressive cartilage loss. Other features of the model include dactylitis and nail changes, symptoms characteristic of psoriatic arthritis (25). This model provides a tool for studying the mechanisms of joint ankylosis with potential relevance to the human spondyloarthropathies. In this report, we evaluate the effects of BMP signaling on initiation and progression of arthritis by gene transfer of noggin, a BMP antagonist.

\section{Results}

Distinct BMPs are expressed in ankylosing enthesitis. We used immunohistochemistry to identify specific BMPs, known to be involved in embryonic endochondral bone formation, in ankylosing enthesitis (Figure 1). The disease process was characterized by inflammation and proliferation at the enthesis, followed by cell differentiation into chondroblasts, prehypertrophic chondrocytes, and hypertrophic chondrocytes (24-27) (Supplemental Figure 1; supplemental material available online with this article; doi:10.1172/JCI23738DS1). The cartilage was progressively replaced by bone, which eventually led to joint ankylosis. Different BMPs were detected in distinct stages of ankylosing enthesitis as indicated by cell morphology (Figure 1). BMP2 was seen in spindle-shaped fibroblast-like cells in the proliferative zones as well as in more rounded chondroblasts in early disease stages (Figure 1, A and B). BMP2 was absent in prehypertrophic and hypertrophic chondrocytes (data not shown). In contrast, in later stages, BMP7 was largely restricted to prehypertrophic chondrocytes (Figure 1, D and E). BMP6 was found in hypertrophic chondrocytes (Figure 1, G and $\mathrm{H}$ ).

Noggin gene transfer inhibits the onset and progression of spontaneous arthritis. We used intramuscular injections of plasmids that encode noggin cDNA under the control of a cytomegalovirus promoter to evaluate ubiquitous suppression of BMP signaling. Injection of $300 \mu \mathrm{g}$ and $30 \mu \mathrm{g}$ pcDNA3.1+noggin resulted in a dose-dependent increase in local mRNA expression (Figure 2, A and B). In the RT-negative control samples, only very discrete amounts of residual noggin cDNA were detected after injection of $300 \mu \mathrm{g}$ pcDNA3.1+noggin. Protein was detectable in muscle and serum
(Figure 2C). Injection of 300- $\mu \mathrm{g}$ pcDNA3.1+ empty vector into the contralateral muscle did not result in locally or systemically detectable noggin. We chose to repeat injections every 3 weeks to ensure gene expression during the course of the experiment (28, 29). To ascertain that repeated injections of noggin cDNA did not result in the formation of antibodies that could block its effect, mice were injected 4 times with $300 \mu \mathrm{g}$ of pcDNA3.1+noggin at 3 -week intervals. After 15 weeks, no specific antibodies were found in their serum (data not shown).

We studied the effect of BMP inhibition on clinical incidence and severity of spontaneous arthritis. Male mice were caged together at the age of 12 weeks and monitored for clinical signs of arthritis until the age of 25 weeks. Animals were treated every 3 weeks (weeks 12, 15, 18, and 21) with plasmid injections (either $300 \mu \mathrm{g}$ pcDNA3.1+noggin, $30 \mu \mathrm{g}$ pcDNA3.1+noggin, or similar amounts of empty pcDNA3.1+). Injections of both $300 \mu \mathrm{g}$ and 30 $\mu \mathrm{g}$ of noggin cDNA reduced the incidence of arthritis as compared with injections of empty vector controls (Gehan-Wilcoxon test, $P<0.05$ ) (Figure 2D). Similarly, pcDNA3.1+noggin reduced severity of arthritis as compared with empty vector (Mann-Whitney $U$ test; $P<0.05$ ) (Figure $2 \mathrm{E}$ ). The effect of noggin treatment on clinical severity during the course of the disease was evaluated by calculating the area under the curve of the clinical severity score (Figure 2F). Both noggin treatment groups $(300 \mu \mathrm{g}$ and $30 \mu \mathrm{g}$ plasmids) showed a reduction in time-integrated clinical severity scores (Mann-Whitney $U$ test, $P<0.05$ ). The effect was dose dependent. We noticed a slower disease onset and slightly reduced severity in the group that was treated with $300 \mu \mathrm{g}$ empty vector as compared with the group treated with $30 \mu \mathrm{g}$ empty vector. Yet this was not statistically significant. However, to exclude that the effect of pcDNA3.1+noggin treatment was due to nonspecific exogenous protein expression by gene transfer, additional mice were treated with pcDNA3.1+ coding for an irrelevant secreted protein (mouse Wnt5a with a mutated hydrophilic domain). No effect on arthritis was found (data not shown).

The effect of BMP inhibition as a therapeutic strategy was evaluated by pcDNA3.1+noggin or empty vector administration (300 $\mu \mathrm{g})$ after symptoms of arthritis had developed. In a first set of experiments, individual mice were injected immediately after the 
first symptoms (toe swelling or stiffness) were recognized. Mice were observed further for 3 weeks. Noggin plasmid treatment significantly reduced clinical disease progression, measured as the difference in clinical score between the beginning and end of the experiment, as compared with empty vector (Figure 3A) (MannWhitney $U$ test, $P<0.05$ ). In the noggin-treated group, 0 out of 6 mice showed disease progression (Figure 3B), and in 2 mice, improvement of arthritis was seen after treatment. In contrast, in the control group, disease progression was seen in 4 out of 8 mice (Figure 3B). In another set of experiments, individual mice were treated 10 days after the first onset of symptoms and further monitored for 3 weeks. Noggin gene therapy resulted in a significant reduction in clinical disease progression as compared with empty vector treatment (Figure 3C) (Mann-Whitney $U$ test, $P<0.05$ ). Of 9 mice, 4 in the noggin-treated group showed signs of clinical improvement, and in 1 animal, the clinical score increased (Figure 4D). In contrast, in the control group, 4 out of 8 mice showed an increase in clinical severity and none of the mice showed improvement of arthritis (Figure 4D).

Noggin modulates endochondral bone formation in ankylosing enthesitis. We studied the effect of exogenous noggin therapy on pathological bone formation by histological analysis in both the preventive and therapeutic experiments described above. Injection of pcDNA3.1+noggin $(300 \mu \mathrm{g})$ every 3 weeks from the age of 12 weeks onward resulted in a significant difference in histological disease severity score as compared with injection of empty vector (Figure 4A) (Mann-Whitney $U$ test, $P<0.05$ ).

We analyzed the effect of noggin gene transfer in this experiment on different stages of endochondral bone formation (Table 1). In the pcDNA3.1+noggin-treated group, 5 out of 8 animals remained unaffected. Cell proliferation was seen in 3 out of 8 mice, but progression to cartilage formation was demonstrated in only 1 animal. Microscopic examination did not reveal bone formation or ankylosis. In contrast, 6 out of 8 animals in the control group were affected. Proliferation of enthesial cells was seen in all affected animals. Four animals showed signs of cartilage formation, and in 1 animal we found bone formation leading to ankylosis.

We also evaluated histological disease severity when noggin cDNA was administered after the first appearance of symptoms. Noggin treatment ( $300 \mu$ g plasmid) resulted in a significantly lower disease severity score as compared with empty vector treatment (Mann-Whitney $U$ test, $P<0.05$ ) (Figure 4B). All animals from both groups showed positive histopathological findings (Table 1). In

\section{Figure 2}

the pcDNA3.1+noggin-treated group, 5 out of 6 animals showed signs of cell proliferation, but only 2 out of 6 showed ectopic cartilage and bone formation. Ankylosis was documented in 1 animal. In contrast, 8 out of 8 animals from the control group showed cell proliferation, 6 out of 8 showed cartilage and bone formation, and 3 out of 8 showed joint ankylosis. Together, these observations suggest that suppression of BMP signaling by noggin can block early stages of pathological endochondral bone formation.

Noggin gene transfer affects proliferation of progenitor cells. We studied the target cell population for BMP signaling in ankylosing enthesitis (Figure 5). Immunofluorescent staining for nuclear phosphorylated
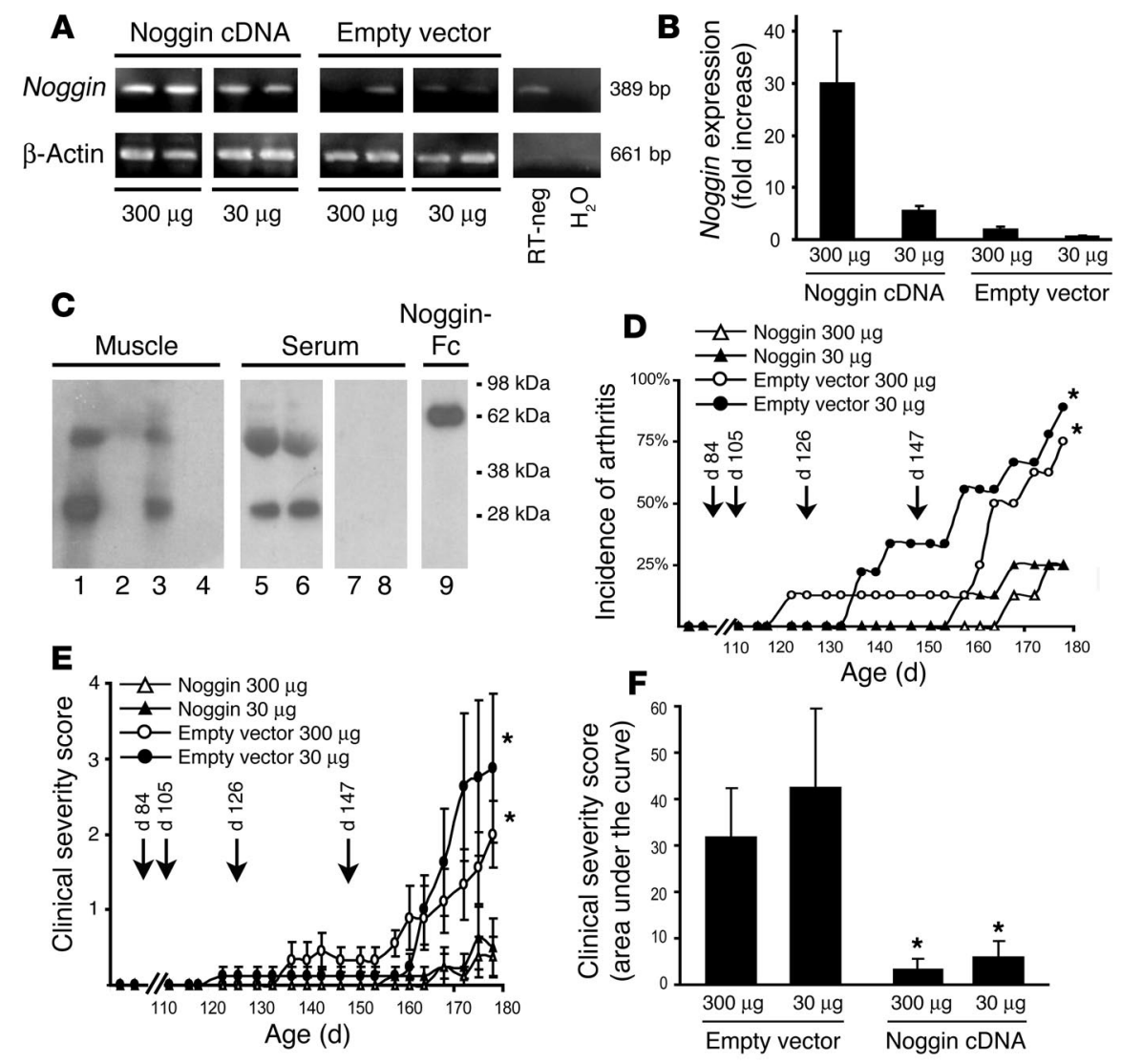

Noggin gene transfer prevents arthritis. (A) RT-PCR demonstrating enhanced expression of noggin 72 hours after cDNA gene transfer $(300 \mu \mathrm{g}$ and $30 \mu \mathrm{g})$. Little endogenous noggin is found. The RT-negative control (RT-neg) obtained from muscle injected with $300 \mu \mathrm{g}$ pcDNA3.1+noggin shows that the RNA extraction procedure successfully removed most of the potentially contaminating cDNA. (B) Real-time PCR analysis of noggin expression 72 hours after cDNA gene transfer. Mean \pm SD from 2 samples. (C) Immunoprecipitation and Western blot demonstrating expression of noggin 72 hours after intramuscular plasmid cDNA gene transfer. A 26-kDa band corresponding to noggin was found in 2 different mice after gene transfer in the injected tibialis anterior muscle (right muscle, lanes 1 and 3 ) but was absent in contralateral control muscle (left muscle, lanes 2 and 4). Noggin was also detected in the serum of both noggin plasmid-injected mice (lanes 5 and 6 ) but not in control mice (lanes 7 and 8). Lane 8 shows a positive control using recombinant noggin-Fc. (D) Noggin gene transfer significantly reduced incidence of spontaneous arthritis as compared with empty vector-treated animals. ${ }^{\star}$ Gehan-Wilcoxon test, $P<0.05$. (E) Noggin gene transfer $(300 \mu \mathrm{g}$ or $30 \mu \mathrm{g})$ significantly reduced severity of spontaneous arthritis as compared with that in empty vector-treated animals. ${ }^{*}$ Mann-Whitney $U$ test; $P<0.05$ at week 25. (F) Noggin gene transfer significantly reduced time-integrated clinical severity, expressed as area under the curve, as compared with that in control animals. *Mann-Whitney $U$ test; $P<0.05$. (D and E) Data are shown as mean \pm SEM; $n=8$ or 9 mice per group. 

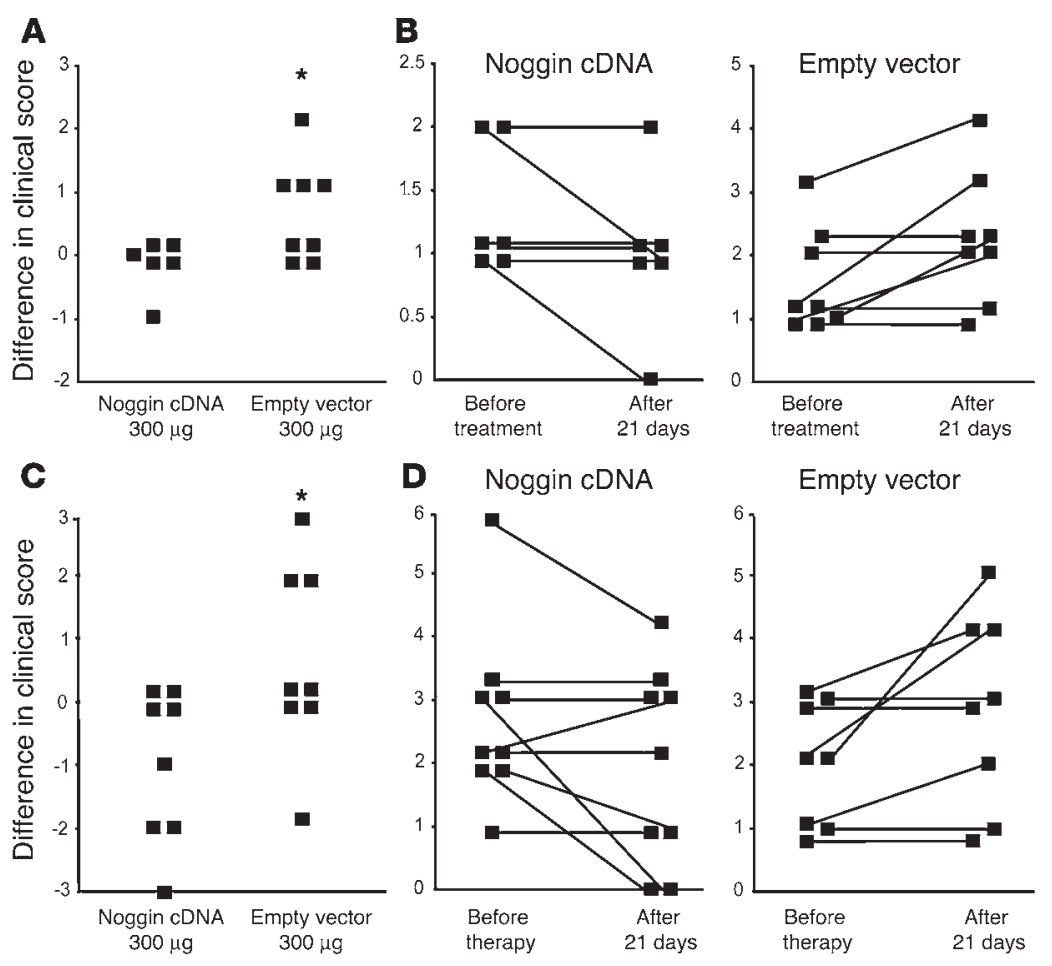

\section{Figure 3}

Noggin gene transfer influenced severity of established arthritis. DBA/1 mice were treated with noggin cDNA injection or empty vector immediately $(\mathbf{A}-\mathbf{B})$ or 10 days (C-D) after the first signs of arthritis had appeared. Disease progression, defined as the difference in clinical score between the beginning and the end of the experiment, was significantly reduced after noggin gene transfer as compared with that in empty vector controls. *Mann-Whitney $U$ test; $P<0.05$, 3 weeks after injection. In $\mathbf{B}$ and $\mathbf{D}$, individual clinical scores before and after treatment are shown.

affected joints. No statistically meaningful differences were seen in BMP7 and IFN- $\gamma$ expression.

Active BMP signaling in human enthesial biopsies. To specifically test whether our findings in the mouse are relevant to new cartilage and bone formation in human disease, we studied activation of BMP signaling in enthesial biopsies obtained from Achilles tendons of spondyloarthropathy patients (Figure 7A). Histological analysis revealed progenitor cell proliferation and cartilage formation strikingly similar to that in the mouse model (Figure 7, B and C). Activation of BMP signaling was apparent in the proliferating and differentiating cell population as revealed by the presence of nuclear phosphorylated-

smad1/5, a marker of active BMP signaling, showed positive nuclei in the enthesial fibroblast-like cells and in cells showing early chondrogenic differentiation (Figure 5A). Phosphorylated-smad1/5 was not present in mature or hypertrophic chondrocytes.

To confirm that noggin gene transfer was inhibiting BMP signaling in affected joints, 3 different sets of interphalangeal joints were dissected ( 5 joints per group): normal joints, arthritic joints, and arthritic joints 6 days after gene transfer. The presence of phosphorylated-smad1/5 molecules was determined by Western blot and digital image analysis. Treatment with 300 $\mu \mathrm{g}$ pcDNA3.1+noggin resulted in a significant reduction in phosphorylated-smad1/5 6 days after treatment as compared with that in arthritic but nontreated joints (Kruskal-Wallis test, $P<0.05$; Mann-Whitney $U$ test, $P<0.05$ ) (Figure 5B).

We hypothesized that BMPs were stimulating proliferation of enthesial progenitors before allowing cells to undergo chondrocytic differentiation. We therefore identified the target cell population for BMP signaling by double staining for proliferating cell nuclear antigen (PCNA), a marker of cell proliferation, and phosphorylated smad1/5 (Figure 5C). Colocalization was found in many but not all enthesial cells.

We further tested whether noggin gene transfer affected the local expression of other molecules that are probably involved in ankylosing enthesitis, such as PCNA (proliferation) and sexdetermining region Y-box 9 (Sox9) (chondrogenesis) (Figure 6A), proinflammatory cytokines (TNF- $\alpha$ and IFN- $\gamma$ ) (Figure 6B), and BMP2, BMP6, and BMP7 (Figure 6C). Normal, affected, and arthritic joints 6 days after noggin gene therapy were tested. Significant differences in affected toes between normal and treated animals were shown for PCNA and BMP2 (Kruskal-Wallis $P<0.05$; Mann-Whitney $U$ test, $P<0.05)$. Similar trends were seen in expression levels of TNF- $\alpha$ and Sox 9 , but this was not confirmed statistically. BMP6 was significantly downregulated in treated samples versus untreated smad1/5 (Figure 7D). As in the mouse model, in later stages, this staining was negative (Figure 7D). Double staining for PCNA and phosphorylated smad $1 / 5$ reflected our observations in mice (Figure 7, E-G). Immunoreactivity for BMP2, BMP7, and BMP6 was recognized in proliferating spindle-shaped cells and in prehypertrophic and mature chondrocytes, respectively (Supplemental Figure 2).

\section{Discussion}

In this study, we demonstrate that BMP signaling affects both the initiation and the progression of ankylosis in a model of anky-
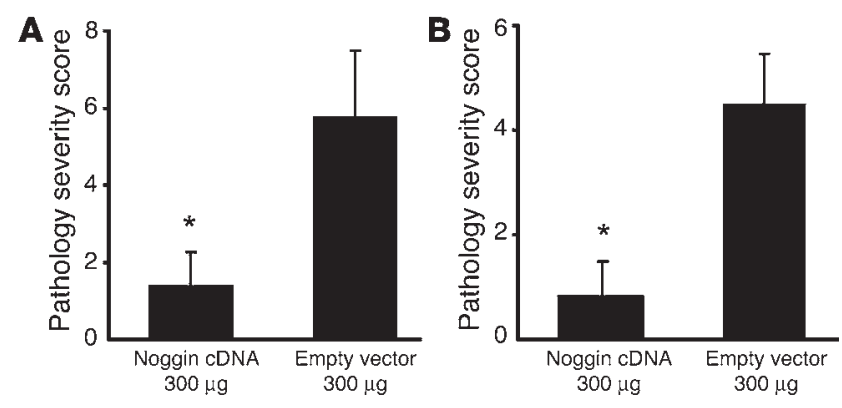

\section{Figure 4}

Noggin gene transfer ameliorated the pathological cascade in ankylosing enthesitis. Noggin or empty vector control gene transfer was performed either as a preventive strategy $(\mathbf{A})$ or as a therapeutic strategy immediately after the first symptoms appeared (B). Noggin gene transfer significantly reduced pathological disease severity in treated animals as compared with empty vector-treated controls. *Mann-Whitney $U$ test, $P<0.05$. Data are shown as mean \pm SEM of the cumulative score of all interphalangeal and metacarpophalangeal joints from the hind feet; each group consisted of 8 mice except the noggin cDNAtreated group in $\mathbf{B}(n=6)$. 
Table 1

Effect of noggin gene transfer on different features of ankylosing enthesitis

\begin{tabular}{lccccc}
\hline & Normal & Proliferation & $\begin{array}{c}\text { Cartilage } \\
\text { formation }\end{array}$ & $\begin{array}{c}\text { Bone } \\
\text { formation }\end{array}$ & $\begin{array}{c}\text { Joint } \\
\text { ankylosis }\end{array}$ \\
$\begin{array}{l}\text { Prevention } \\
\quad \text { Empty vector }\end{array}$ & $2 / 8(25)$ & $6 / 8(75)$ & $4 / 8(50)$ & $1 / 8(12)$ & $1 / 8(12)$ \\
$\quad$ Noggin & $5 / 8(62)$ & $3 / 8(37)$ & $1 / 8(0.12)$ & $0 / 8(0)$ & $0 / 8(0)$ \\
$\begin{array}{l}\text { Therapy } \\
\quad \text { Empty vector }\end{array}$ & $0 / 8(0)$ & $8 / 8(100)$ & $6 / 8(75)$ & $6 / 8(75)$ & $3 / 8(50)$ \\
$\quad$ Noggin & $0 / 6(17)$ & $5 / 6(83)$ & $2 / 6(33)$ & $2 / 6(33)$ & $1 / 6(17)$ \\
\hline
\end{tabular}
the total number of animals in the group. Percentages are shown in parentheses.
Data are shown as the number of animals exhibiting a specific trait as compared with

in this model, in contrast to other models of ankylosis (35), highlight its usefulness for mechanistic studies in the process of ankylosis in spondyloarthropathy. As no animal model completely mimics human disease, different questions can be raised regarding the relevance of this model to human spondyloarthropathy. First, the absence of spine pathology in this model can at least partially be explained by differences in weight bearing between rodents and humans. So far, there are no indications that ectopic bone formation in spine and in peripheral joints is distinct. Both processes mimic predominantly endochondral bone formation $(5,36)$. Second, the inflammatory reaction associated with the proliferative and metaplastic process at the enthesis is still poorly defined. We have demonstrated that dactylitis or local inflammation losing enthesitis and spondyloarthropathy. BMP signaling has been studied in a mouse model of degenerative joint disease (30). Ectopic bone formation in this disorder may be a consequence of structural damage and is likely to be part of the body's response to local injury, either as an attempt to increase joint stability or as a secondary effect of growth factors trying to preserve joint homeostasis (31). Our data provide evidence that deregulation of specific embryonic signaling pathways can also contribute to pathology. Indeed, several arguments indicate that the process of enthesial bone formation leading to ankylosis, as seen in spondyloarthropathies and in this model, is different from osteophytes in osteoarthritis. First, progressive ankylosis in both spine and peripheral joints is a major contributor to disability. Second, ankylosing enthesitis presents frequently as clinically symptomatic arthritis. Third, in contrast to osteoarthritis, heterotopic endochondral bone formation takes place in tissue that is considered one of the primary disease targets $(3,5,32)$. Therefore, inhibition of ankylosis may be an important and specific therapeutic target in spondyloarthropathy $(11,33)$.

To address the mechanisms driving joint ankylosis, we have used a spontaneous mouse model of arthritis. Although it was originally reported as another model of rheumatoid arthritis (23), subsequent studies involving microscopic analysis have clearly demonstrated that this model is characterized by ankylosing enthesitis $(25,27)$ rather than destructive synovitis. This model is particularly attractive since arthritis occurs in association with environmental factors such as stress that have also been implicated in spondyloarthropathy (24, 34). It also shows specific features of psoriatic arthritis (25) that have not been reported in other models of arthritis. The high incidence and reproducibility of arthritis
A
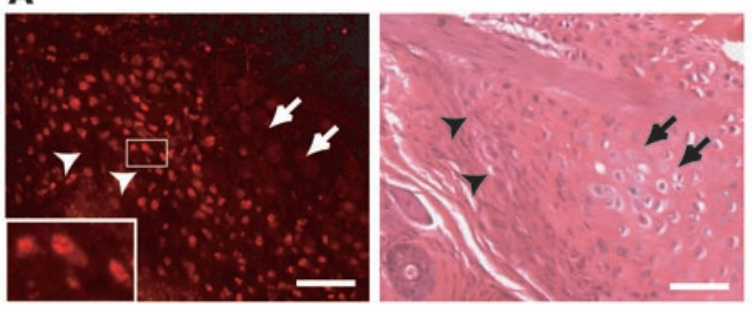

B Normal joints

Affected joints
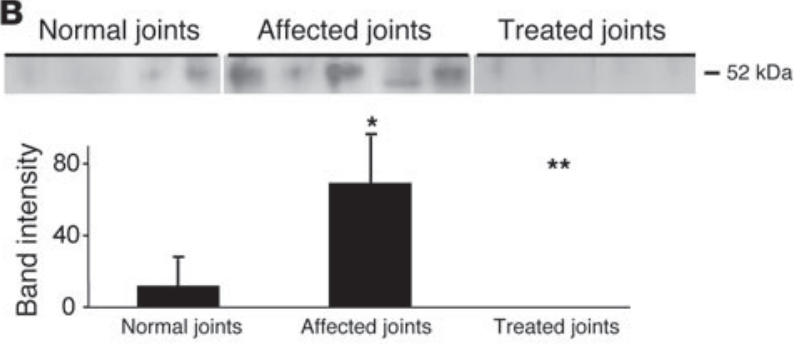

at the enthesis, characterized by the presence of neutrophils and some mononuclear cells, precedes or coincides with connective tissue proliferation and differentiation (25). Remarkably, Nordling et al. demonstrated that anti-idiotypic anticollagen type II antibody treatment may prevent the onset of arthritis (37). Autoimmunity toward components of fibrocartilage has been proposed by different authors as directly relevant for the development of spondyloarthropathy $(5,38)$. Autoantibodies toward type II collagen have also been demonstrated in spondyloarthropathy patients (39). Interestingly, we have also shown that inhibition of IFN- $\gamma$ delays the onset of disease (26). However, in contrast to the effect of noggin, no therapeutic effect of anti-IFN- $\gamma$ strategies was seen once symptoms had occurred (26). The finding that the disease process is

\section{Figure 5}

Noggin inhibited BMP signaling in spontaneous arthritis. (A) Immunofluorescent staining for phosphorylated smad1/5/8 demonstrating positive nuclei (red staining, arrowheads; detail in inset) in the zone of proliferating cells. The area corresponding to hypertrophic chondrocytes is negative (arrows). A representative, adjacent section stained with H\&E is also shown. (B) Phosphorylation of smad1/5/8 was increased in arthritic as compared with healthy interphalangeal joints and was inhibited by noggin gene transfer as shown by semiquantitative Western blot $(n=5$ animals per group, Kruskal-Wallis test $P<0.05$; Mann-Whitney $U$ test: ${ }^{*} P<0.05$ versus normal joints; ${ }^{* *} P<0.05$ versus untreated affected joints). (C) Double immunofluorescense staining for phosphorylated smad1/5 (red) and PCNA (green) demonstrating nuclear colocalization (yellow in overlay). Scale bars: $50 \mu \mathrm{m}$. 

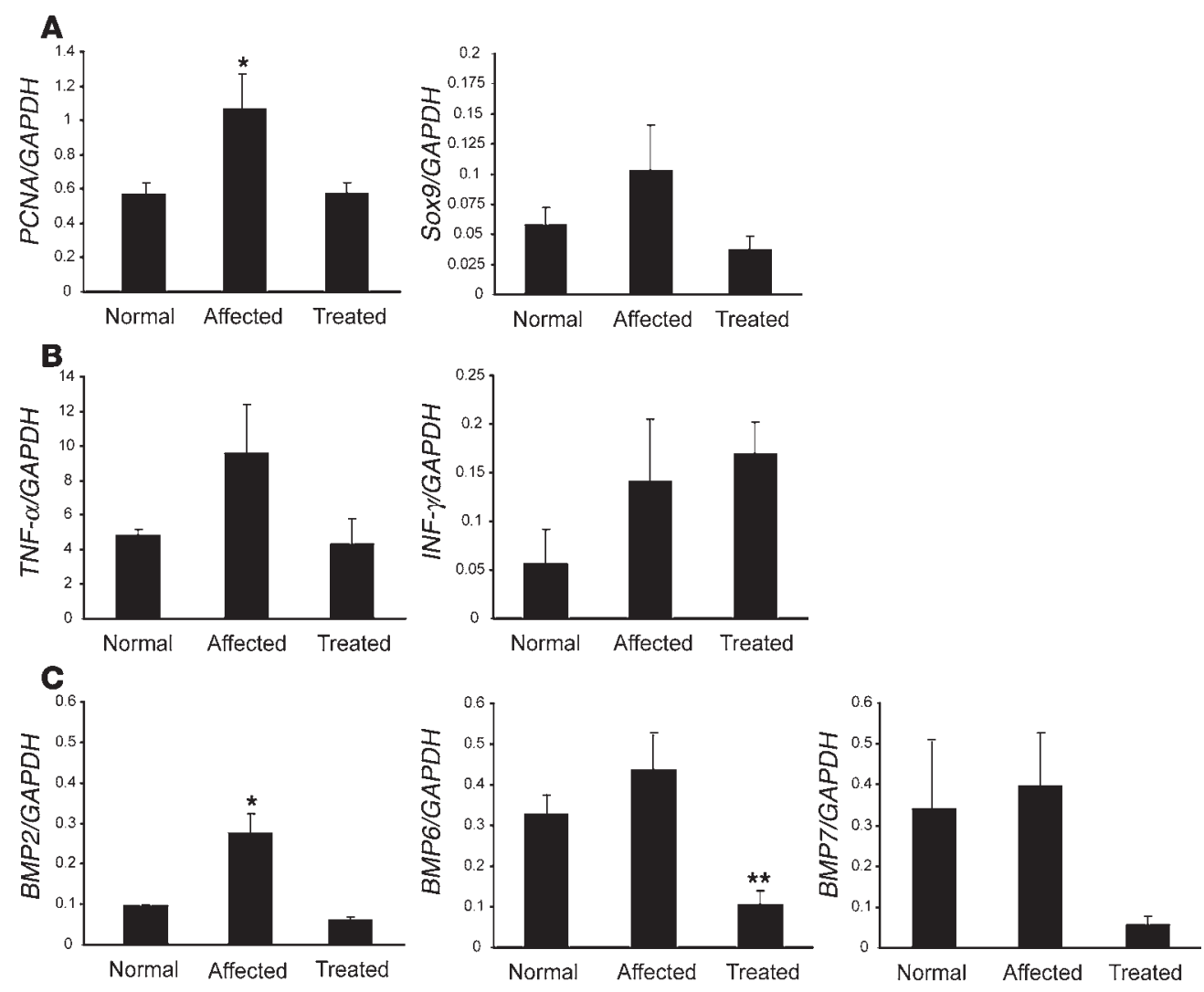

\section{Figure 6}

Systemic noggin gene transfer and gene expression in interphalangeal joints. Cell cycle and differentiation markers (A), proinflammatory cytokines (B), and BMPs (C) were studied by real-time PCR analysis. Data are expressed as amounts relative to the expression of GAPDH. $n=5$ animals per group. *Kruskal-Wallis test, $P<0.05$; Mann-Whitney $U$ test, $P<0.05$ for affected as compared with normal and treated animals. **Kruskal-Wallis test, $P<0.05$; Mann-Whitney $U$ test, $P<0.05$ for treated as compared with affected animals. The $y$ axes indicate expression levels relative to the expression of GAPDH. apparently $\mathrm{T}$ cell independent seems to be in contrast with the proposed role for T cells in both psoriatic arthritis (40) and ankylosing spondylitis (5). In these experiments, however, $\mathrm{T}$ cell receptors $\alpha \beta^{-/-}$and $\gamma \delta^{-/-}$were used separately. Therefore, a role for T cells in the development of autoimmunity toward fibrocartilage components in this model cannot be excluded. Taken together, all these data support the concept of juxtaposed stages in the disease that are coupled but partially independent. Our model therefore seems representative of the process of ankylosis in spondyloarthropathy, which can be considered as a specific therapeutic target.

The somewhat surprising effects in the early stages of the disease highlight the importance of BMP signaling. This pathway is an essential part of the complex network regulating skeletal development (14-18). Endochondral bone formation is initiated by mesenchymal cell condensations. Cells within these condensations undergo chondrogenic differentiation, progressively acquiring the phenotypes of proliferating, prehypertrophic, and hypertrophic chondrocytes. In the later stages, the cartilaginous elements are replaced by bone (13). Studies in developmental models suggest that fine-tuned balances between BMPs and inhibitors such as noggin appear to influence different stages of endochondral bone formation. Retroviral over- and misexpression of noggin in the developing chick limb revealed a dual role for BMP signaling in early stages (41). Both cell condensation and differentiation of chondroprogenitor cells into chondrocytes were inhibited effectively, depending on the time of infection.

Various challenges regarding the role of BMPs in ankylosing enthesitis remain. First, understanding of the relative contribution of specific BMPs and inhibitors to the pathological cascade needs to be refined. Our data suggest an early role for BMP2 and later involvement of BMP6 and BMP7. The presence of additional ligands has not been studied. However, regardless of their presence, noggin has been demonstrated as an antagonist of the BMP2/4, the BMP5/6/7, and the growth and differentiation factor $5 / 6 / 7$ groups (42). Therefore, noggin overexpression is likely to change the overall balance in BMP signaling in our model. Second, the identification of factors leading to the activation of the BMP-signaling pathway is of critical importance to understanding the links between inflammation and bone formation, but the exact mechanism remains unknown. We and other groups have demonstrated that specific BMPs, including BMP2 and BMP6, are upregulated by proinflammatory cytokines in cell populations obtained from the joint, providing circumstantial evidence that inflammatory changes and BMP expression are linked (43-45). Other explanations include increased expression of BMP receptors or BMP-signaling enhancers (46), through genetic or environmental factors, that lead to enhanced activation of the smad pathway.

It is well known that the biological response to BMPs is dependent on the target tissue (19). Injection of recombinant BMP2 into the knee joints of mice resulted in osteophyte formation in areas adjacent to the bone and did not result in cartilage formation in the synovium itself (47). Adenoviral infection of periost with BMP2 in vivo stimulated endochondral bone formation at the application site (48).

In summary, our findings support the concept that BMP signaling is an attractive therapeutic target for achieving disease modification in spondyloarthropathy. Symptom control by inhibition of inflammation may not be sufficient to stop the structural progression of disease and the resulting disability. Therefore, specific molecular targets involved in cartilage and bone forma- 

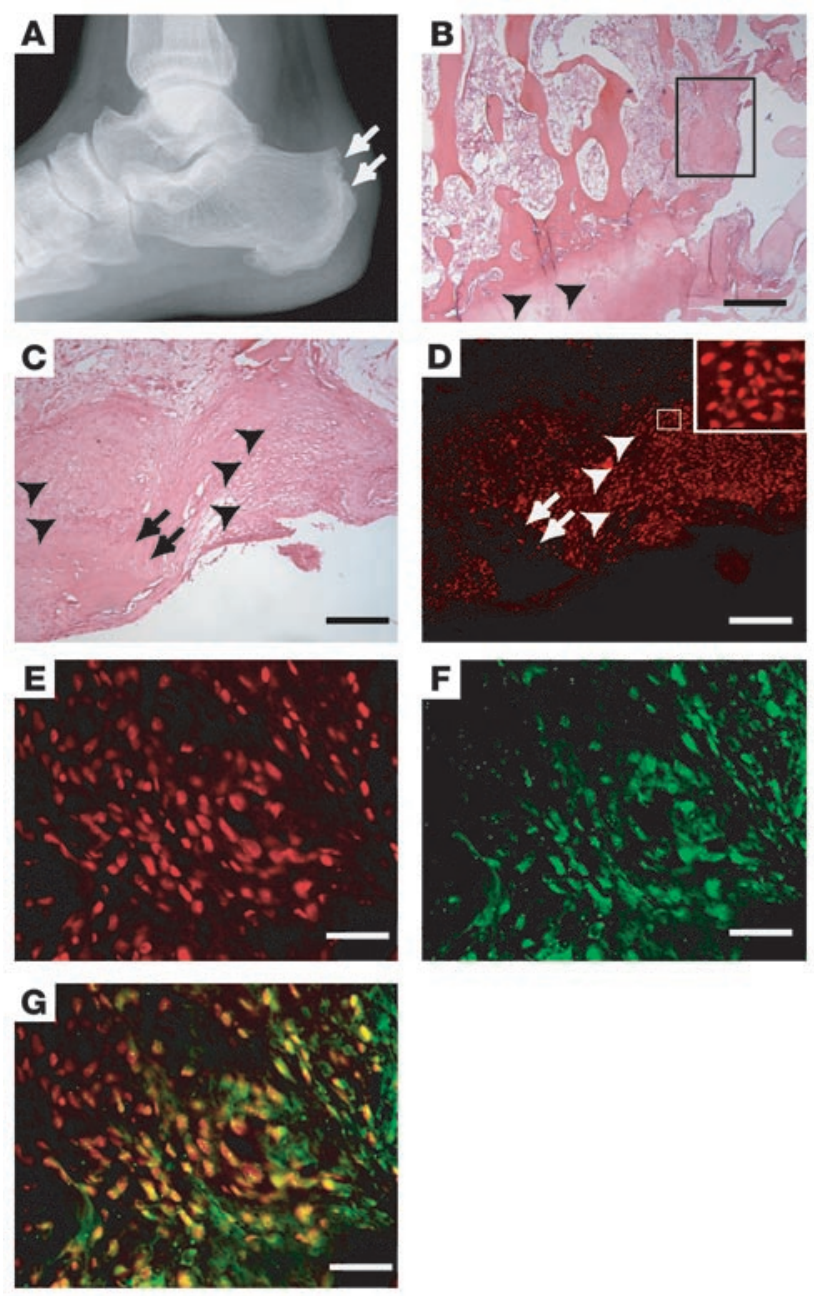

tion, including the BMPs and their transduction machinery, may provide a complementary or alternative therapeutic approach in patients with spondyloarthropathies. In the complex network of cell differentiation and lineage commitment, molecular networks may be crucial, and single-gene approaches to treating diseases may not be sufficient.

\section{Methods}

Animal experiments. Three sets of experiments were performed. First, male DBA/1 mice (Jackson Laboratory or Janvier) from different litters were mixed and caged together in groups of 4 to 6 mice at the age of 12 weeks. Full-length mouse noggin cDNA (a gift of R. Harland, University of California, Berkeley, California, USA) was cloned into pcDNA3.1+ vector (Invitrogen Corp.). Either 300 or $30 \mu \mathrm{g}$ of pcDNA3.1+noggin or pcDNA3. $1^{+}$was injected into the tibialis anterior muscles at weeks 12 , 15,18 , and 21 . Animals were sacrificed at 25 weeks. Second, after the first symptoms appeared in each mouse, pcDNA3.1+noggin $(300 \mu \mathrm{g})$ or empty vector was injected immediately. Third, animals with clinical signs of arthritis were treated with pcDNA3.1+noggin $(300 \mu \mathrm{g})$ or empty vector 10 days after the first symptoms were noted. In these experiments, individual mice were killed 3 weeks after injection of plasmid cDNA. The Ethics Committee for Animal Research (Katholieke Universiteit Leuven) approved all animal experiments. All experiments and analyses were run in a randomized and blinded fashion.

\section{Figure 7}

BMP signaling in human enthesitis in spondyloarthropathy. (A) X-ray image showing irregular borders and bony outgrowth (arrows) at the Achilles enthesis. (B) Overview of an enthesial biopsy stained with $\mathrm{H} \& \mathrm{E}$, showing a normal attachment zone (arrowheads) and new tissue formation (boxed area). (C) Detail of boxed area in $\mathbf{B}$ showing proliferation (arrowheads) and cartilage formation (arrows). (D) Immunofluorescent staining for phosphorylated-smad $1 / 5 / 8$ showing positive proliferating cells (arrowheads, detail in inset) and negative chondrocytes (arrows). (E-G) Double immunofluorescence for phosphorylatedsmad1/5 (E), PCNA (F), and overlay (G). Scale bars: B, $800 \mu \mathrm{m}$; C and D, $200 \mu \mathrm{m} ; \mathbf{E}$ and $\mathbf{G}, 50 \mu \mathrm{m}$.

Mice were scored twice a week for clinical signs of arthritis as follows $(26,27)$ : 0 , no symptoms; 1 , redness and swelling in one toe; 2 , redness and swelling in more than one toe; 3 , toe stiffness; and 4, deformity or ankle involvement. Both hind paws were evaluated, resulting in a maximum score of 8 . For histological examination, hind paw forefeet were formalin-fixed, decalcified using Decal (Serva), paraffin embedded, and cut in a transverse plane. A histological score was developed previously $(25,26)$ : 1 , inflammatory cell infiltration; 2 , enthesial fibroblast-like cell proliferation; 3 , cartilage formation; 4, bone formation; and 5, ankylosis. In each mouse, a cumulative score for all interphalangeal and metatarsophalangeal joints of the 2 hind limbs was calculated by summation of the individual joint scores. Both clinical and pathological scores were found to be consistent and reproducible with little intra- and interobserver variability.

Human materials. Enthesial biopsies were obtained during surgery for refractory enthesitis. The Ethics Committee for Human Medical Research (Katholieke Universiteit Leuven) approved all procedures, and patient informed consent was obtained.

Immunohistochemistry and immunofluorescence. For immunohistochemistry and immunofluorescence, sections were quenched with $3 \% \mathrm{H}_{2} \mathrm{O}_{2} / \mathrm{H}_{2} \mathrm{O}$ or $50 \mathrm{mM} \mathrm{NH}_{4} \mathrm{Cl}$ respectively and preincubated with donkey serum $(20 \%$ in Tris-buffered saline [TBS]). Sections were incubated with polyclonal primary antibodies against BMP2 (Pfizer) $(5 \mu \mathrm{g} / \mathrm{ml})$, BMP7 (Pfizer) $(10 \mu \mathrm{g} /$ ml), BMP6 (Santa Cruz Biotechnology Inc.) $(10 \mu \mathrm{g} / \mathrm{ml})$, phosphorylated smad1/5 (PS1) (P. Ten Dijke, University of Leiden, Leiden, The Netherlands) $(1 / 100)$ overnight at $4{ }^{\circ} \mathrm{C}$. Anti-BMP2 antibody was raised against peptide sequence Ac-REKRQAKHKARKRLKSSC-NH2. BLAST protein analysis (http://www.ncbi.nlm.nih.gov/BLAST) showed that this peptide is specific for BMP2 and is conserved across species (human, mouse, rat, xenopus). Anti-BMP7 antibody was raised against peptide sequence Ac-TGSKQRSQNRSKTPKNC-NH2. This peptide sequence is specific for human BMP7, as shown by BLAST protein analysis. The mouse and rat BMP7 sequence showed 1 amino acid difference ( $G$ instead of $S$ in position 3). Anti-BMP6 was raised against a peptide sequence from the aminoterminus of human BMP6. Antiphosphorylated smad1/5 antibody recognizing the phosphorylated C-tail in Smad1 was generated by injection of peptide KKK-NPISSVS containing $2 \mathrm{C}$ terminal phosphoserine residues coupled to keyhole limpet hemocyanin (49). Negative controls were performed with species-specific IgG (Jackson ImmunoResearch Laboratories Inc.) or preincubation of the antibodies with blocking peptides or proteins. After washing and a second blocking step, sections were incubated with horseradish peroxidase-conjugated secondary antibodies (Jackson ImmunoResearch Laboratories Inc.) (1/100). For immunofluoresence, secondary antibodies were Cy3-conjugated goat anti-rabbit antibody (1/100 dilution) or Cy2-conjugated anti-goat antibody (1/100 dilution) (Jackson ImmunoResearch Laboratories Inc.).

Western blot analysis. For phosphorylated smad1/5, interphalangeal joints were dissected, frozen, thawed twice, and stored in $6 \mathrm{M}$ urea/10 mM 
Tris/pH 7.8. Five-hundred ng protein, diluted in $4 \times$ NuPAGE-LDS buffer (Invitrogen Corp.) was analyzed under reducing conditions ( $2 \%$ mercaptoethanol and 0.1 M DTT) in NuPAGE-MES-SDS buffer (Invitrogen Corp.). Proteins were transferred onto a PVDF membrane in $0.4 \mathrm{M}$ glycine/ 0.5 M Tris-base/0.01 M SDS and $200 \mathrm{ml} / 1$ methanol. Blots were probed with antibody PS1 (1/2500 in TBS-Triton/3\% milk) and washed with TBS-Triton, and goat anti-rabbit horseradish peroxidase-conjugated secondary antibody (1/5000 in TBS-Triton/3\% milk) (Jackson ImmunoResearch Laboratories Inc.) was added. For visualization, we used SuperSignal Pico Chemiluminescent Substrate (Pierce).

For noggin, tibialis anterior muscles were injected with $300 \mu \mathrm{g}$ pcDNA3.1+noggin or empty vector. After 72 hours, muscles and blood were collected. Muscles were extracted in $1 \mathrm{M}$ guanidium hydrochloride/50 mM Na-acetate overnight. Samples were desalted, lyophilized, and redissolved in 200- $\mu \mathrm{l}$ immunoprecipitation buffer $(150 \mathrm{mM} \mathrm{NaCl} / 1 \%$ NP-40/2 mM EDTA/50 mM NaF/1 mM Na $4 \mathrm{P}_{2} \mathrm{O}_{7} / 20 \mathrm{mM}$ Tris/pH 7.6). Samples and serum were incubated overnight at $4{ }^{\circ} \mathrm{C}$ with $2 \mu \mathrm{g}$ goat anti-noggin antibody. Protein $\mathrm{A} / \mathrm{G}$ sepharose solution (Amersham Biosciences) was used for precipitation. Pellets were dissolved in loading buffer-8 $\mathrm{M}$ urea. Western blot was performed as above with goat anti-noggin polyclonal affinity purified antibody (R\&D Systems) (1/5000) and horseradish peroxidase-conjugated mouse anti-goat (1/20000) (Jackson ImmunoResearch Laboratories Inc.). Recombinant noggin-Fc (R\&D Systems) (10 ng) was used a positive control.

PCR. RNA was isolated from tibialis anterior muscle or interphalangeal joints using Trizol (Invitrogen Corp.) and reverse transcribed (Superscript III; Invitrogen Corp.). For conventional PCR, the following primers were used: $\beta$-actin forward primer 5 '-TGACGGGGTCACCCACACTGTGCCCATCTA-3'; $\beta$-actin reverse primer $5^{\prime}$-CTAGAAGCATTTGCGGTGGACGATG-
GAGGG-3'; noggin forward primer 5'-GCATGGAGCGCTGCCCCAGC-3'; noggin reverse primer $5^{\prime}$-GAGCAGCGAGCGCAGCAGCG-3'. For $\beta$-actin, a 3 -step PCR was performed with an annealing temperature of $60^{\circ} \mathrm{C}$; for $n o g$ gin, a 2-step PCR was performed with an annealing temperature of $72^{\circ} \mathrm{C}$. For real-time PCR, gene expression was studied using Assay-on-Demand primer-probe systems (Applied Biosystems). Expression levels were normalized to GAPDH expression using the comparative threshold cycle method.

Statistics. Comparisons between groups were made by Kruskal-Wallis test and Mann-Whitney $U$ test. For incidence, Gehan-Wilcoxon test was used.

\section{Acknowledgments}

The authors wish to thank Przemko Tylzanowski, Kurt de Vlam, Rene Westhovens, and Malcolm Moos for useful suggestions and critical appraisal of the data; Jenny Peeters and Erik-Jan Ververs for technical assistance; and Giovanni Matricali for enthesial biopsies. This work was supported by grant 0.390 .03 from the Fund for Scientific Research - Flanders. R.J.U. Lories has received an Aspirant fellowship and is currently a postdoctoral fellow; both awards are from the Fund for Scientific Research - Flanders.

Received for publication October 26, 2004, and accepted in revised form April 12, 2005.

Address correspondence to: Frank P. Luyten, Department of Rheumatology, University Hospitals Leuven, Herestraat 49, B-3000 Leuven, Belgium. Phone: 32-16-346341; Fax: 32-16-346200; E-mail: Frank.Luyten@uz.kuleuven.ac.be.

Frank P. Luyten is the senior author.
1. Saraux, A., et al. 1999. Prevalence of rheumatoid arthritis and spondyloarthropathy in Brittany, France. J. Rheumatol. 26:2622-2627.

2. Wright, V. 1978. Seronegative polyarthritis: a unified concept. Arthritis Rheum. 21:619-633.

3. Ball, J. 1971. Enthesopathy of rheumatoid and ankylosing spondylitis. Ann. Rheum. Dis. 30:213-223.

4. Benjamin, M., and McGonagle, D. 2001. The anatomical basis for disease localisation in seronegative spondyloarthropathy at entheses and related sites. J. Anat. 199:503-526.

5. Francois, R.J., Braun, J., and Khan, M.A. 2001. Entheses and enthesitis: a histopathologic review and relevance to spondyloarthritides. Curr. Opin. Rheumatol. 13:255-264.

6. van der Heijde, D., Braun, J., McGonagle, D., and Siegel, J. 2002. Treatment trials in ankylosing spondylitis: current and future considerations. Ann. Rheum. Dis. 61:24-32.

7. Braun, J., et al. 2002. Treatment of active ankylosing spondylitis with infliximab: a randomised controlled multicentre trial. Lancet. 359:1187-1193.

8. Mease, P.J., et al. 2000. Etanercept in the treatment of psoriatic arthritis and psoriasis: a randomised trial. Lancet. 356:385-390.

9. Kong, Y.Y., et al. 1999. Activated T cells regulate bone loss and joint destruction in adjuvant arthritis through osteoprotegerin ligand. Nature. 402:304-309.

10. Marzo-Ortega, H., Emery, P., and McGonagle, D. 2002. The concept of disease modification in spondyloarthropathy. J. Rheumatol. 29:1583-1585.

11. Zhang, X., Aubin, J.E., and Inman, R.D. 2003. Molecular and cellular biology of new bone formation: insights into the ankylosis of ankylosing spondylitis. Curr. Opin. Rheumatol. 15:387-393.

12. Urist, M.R. 1965 . Bone: formation by autoinduction. Science. 150:893-899.

13. Olsen, B.R., Reginato, A.M., and Wang, W.F.
2000. Bone development. Annu. Rev. Cell Dev. Biol. 16:191-220.

14. Kronenberg, H.M. 2003. Developmental regulation of the growth plate. Nature. 423:332-336.

15. Thomas, J.T., et al. 1996. A human chondrodysplasia due to a mutation in a TGF-beta superfamily member. Nat. Genet. 12:315-317.

16. Thomas, J.T., et al. 1997. Disruption of human limb morphogenesis by a dominant negative mutation in CDMP1. Nat. Genet. 17:58-64.

17. Polinkovsky, A., et al. 1997. Mutations in CDMP1 cause autosomal dominant brachydactyly type C. Nat. Genet. 17:18-19.

18. Storm, E.E., et al. 1994. Limb alterations in brachypodism mice due to mutations in a new member of the TGF beta-superfamily. Nature. 368:639-643.

19. Massague, J. 2000. How cells read TGF-beta signals. Nat. Rev. Mol. Cell Biol. 1:169-178.

20. Waite, K.A., and Eng, C. 2003. From developmental disorder to heritable cancer: it's all in the BMP/ TGF-beta family. Nat. Rev. Genet. 4:763-773.

21. Steiling, H., et al. 2003. Fibroblast growth factor receptor signalling is crucial for liver homeostasis and regeneration. Oncogene. 22:4380-4388.

22. Reddi, A.H. 2003. Cartilage morphogenetic proteins: role in joint development, homoeostasis, and regeneration. Ann. Rheum. Dis. 62:73-78.

23. Nordling, C., Karlsson-Parra, A., Jansson, L., Holmdahl, R., and Klareskog, L. 1992. Characterization of a spontaneously occurring arthritis in male DBA/1 mice. Arthritis Rheum. 35:717-722.

24. Holmdahl, R., Jansson, L., Andersson, M., and Jonsson, R. 1992. Genetic, hormonal and behavioural influence on spontaneously developing arthritis in normal mice. Clin. Exp. Immunol. 88:467-472.

25. Lories, R.J., Matthys, P., de Vlam, K., Derese, I., and Luyten, F.P. 2004. Ankylosing enthesitis, dactylitis and onychoperiostitis in a mouse model of psoriatic arthritis. Ann. Rheum. Dis. 63:595-598.
26. Matthys, P., et al. 2003. Dependence on interferongamma for the spontaneous occurrence of arthritis in DBA/1 mice. Arthritis Rheum. 48:2983-2988.

27. Corthay, A., Hansson, A.S., and Holmdahl, R. 2000. $\mathrm{T}$ lymphocytes are not required for the spontaneous development of entheseal ossification leading to marginal ankylosis in the DBA/ 1 mouse. Arthritis Rheum. 43:844-851.

28. Song, X.Y., Gu, M., Jin, W.W., Klinman, D.M., and Wahl, S.M. 1998. Plasmid DNA encoding transforming growth factor- $\beta 1$ suppresses chronic disease in a streptococcal cell wall-induced arthritis model. J. Clin. Invest. 101:2615-2621.

29. Zhang, Z.L., et al. 2003. Intramuscular injection of interleukin-10 plasmid DNA prevented autoimmune diabetes in mice. Acta Pharmacol. Sin. 24:751-756.

30. Scharstuhl, A., Vitters, E.L., van der Kraan, P.M., and van Den Berg, W.B. 2003. Reduction of osteophyte formation and synovial thickening by adenoviral overexpression of transforming growth factor beta/bone morphogenetic protein inhibitors during experimental osteoarthritis. Arthritis Rheum. 48:3442-3451.

31. Sandell, L.J., and Aigner, T. 2001. Cell biology of osteoarthritis. Arthritis Res. 3:107-113.

32. McGonagle, D., Gibbon, W., and Emery, P. 1998. Classification of inflammatory arthritis by enthesitis. Lancet. 352:1137-1140.

33. Dougados, M., et al. 2002. Conventional treatments for ankylosing spondylitis. Ann. Rheum. Dis. 61:40-50.

34. Gooren, L.J.G., Giltay, E.J., van Schaardenburg, D., and Dijkmans, B.A.C. 2000. Gonadal and adrenal sex steroids in ankylosing spondylitis. Rheum. Dis. Clin. North Am. 26:969-987.

35. Weinreich, S., Capkova, J., Hoebe-Hewryk, B., Boog, C., and Ivanyi, P. 1996. Grouped caging predisposes male mice to ankylosing enthesopathy. Ann. Rheum. 
Dis. 55:645-647.

36. Francois, R.J., Gardner, D.L., Degrave, E.J., and Bywaters, E.G. 2000. Histopathologic evidence that sacroiliitis in ankylosing spondylitis is not merely enthesitis. Arthritis Rheum. 43:2011-2024.

37. Nordling, C., Kleinau, S., and Klareskog, L. 1992. Down-regulation of a spontaneous arthritis in male DBA/ 1 mice after administration of monoclonal anti-idiotypic antibodies to a cross-reactive idiotope on anti-collagen antibodies. Immunology. 77:144-146.

38. Maksymowych, W.P. 2000. Ankylosing spondylitisat the interface of bone and cartilage. J. Rheumatol. 27:2295-2301.

39. Tani, Y., Sato, H., and Hukuda, S. 1997. Autoantibodies to collagens in Japanese patients with ankylosing spondylitis. Clin. Exp. Rheumatol. 15:295-297.

40. Hohler, T., and Marker-Hermann, E. 2001. Psoriatic arthritis: clinical aspects, genetics, and the role of T cells. Curr. Opin. Rheumatol. 13:273-279.
41. Pizette, S., and Niswander, L. 2000. BMPs are required at two steps of limb chondrogenesis: Formation of prechondrogenic condensations and their differentiation into chondrocytes. Dev. Biol. 219:237-249

42. Balemans, W., and Van Hul, W. 2002. Extracellular regulation of BMP signaling in vertebrates: a cocktail of modulators. Dev. Biol. 250:231-250.

43. Fowler, M.J., Jr., et al. 1998. Induction of bone morphogenetic protein- 2 by interleukin-1 in human fibroblasts. Biochem. Biophys. Res. Commun. 248:450-453.

44. Lories, R.J.U., Derese, I., Ceuppens, J.L., and Luyten, F.P. 2003. Bone morphogenetic proteins 2 and 6 , expressed in arthritic synovium, are regulated by proinflammatory cytokines and differentially modulate fibroblast-like synoviocyte apoptosis. Arthritis Rheum. 48:2807-2818.

45. Fukui, N., Zhu, Y., Maloney, W.J., Clohisy, J., and Sandell, L.J. 2003. Stimulation of BMP-2 expres- sion by pro-inflammatory cytokines IL-1 and TNFalpha in normal and osteoarthritic chondrocytes. J. Bone Joint Surg. Am. 85(Suppl. 3):59-66.

46. Lin, J., et al. 2005. Kielin/chordin-like protein, a novel enhancer of BMP signaling, attenuates renal fibrotic disease. Nat. Med. 11:387-393.

47. van Beuningen, H.M., Glansbeek, H.L., van der Kraan, P.M., and van Den Berg, W.B. 1998. Differential effects of local application of BMP-2 or TGF-beta 1 on both articular cartilage composition and osteophyte formation. Osteoarthr. Cartil. 6:306-317.

48. Uusitalo, H., et al. 2001. Induction of periosteal callus formation by bone morphogenetic protein-2 employing adenovirus-mediated gene delivery. Matrix Biol. 20:123-127.

49. Persson, U., et al. 1998. The L45 loop in type I receptors for TGF-beta family members is a critical determinant in specifying Smad isoform activation. FEBS Lett. 434:83-87. 